\title{
Capturing and communicating advanced mathematical activity
}

\section{Peter Samuels}

Birmingham City University, UK

\begin{abstract}
Unlike most other subjects, mathematical activity resides almost entirely within the cognitive processes of a mathematics practitioner and is therefore difficult to characterise. Despite recent interest, the nature of advanced mathematical activity remains something of a black box to educational researchers. In addition, the production of advanced mathematical texts, such as theses or journal articles, is often divorced from mathematicians' experiences of creating mathematics which can lead them to a sense of personal alienation from their work. This article proposes four practical techniques for capturing advanced mathematical activity. The timing of the use of these techniques is compared against a model of mathematical creativity and the writing process. The establishment of a new genre for communicating advanced mathematics is proposed which combines the product of the activity with the process of creating it.
\end{abstract}

Keywords: mathematical creativity; advanced mathematical activity; mathematical writing; data capturing techniques; mathematical activity corpus.

\section{Introduction}

Creating mathematics can be an alienating experience for mathematicians. This is because the product of mathematical activity can be far removed from the process by which it is created. Marx (1974, p. 64) described alienation from product as:

The worker is related to the product of his labour as to an alien object. For on this premise it is clear that the more the worker spends himself, the more powerful the alien objective world becomes which he creates over against himself - his inner world - becomes, the less belongs to himself as his own. 
Rowan (1981) applied Marx's theory of alienation to the traditional paradigm of research in the human sciences. I claim that it is even more applicable to mathematics research.

The four stage model of the creative process is widely attributed to Poincaré (1908) who described a four stage process for his own mathematical creativity: preparation, incubation, illumination, and verification. According to Lubart (2001), by 1950 there was considerable agreement that this model describes the creative process in general (Guilford, 1950). Crowley (1977) suggested the application of this model to describing the writing process with writing replacing illumination and revision replacing verification. Other writing process researchers have reclassified preparation and incubation as prewriting, writing as drafting and have added editing after revision. Whilst the four stage model has been criticised for lacking psychological depth by Guilford (1950) in its application to general creativity, and by Flower and Hayes (1981) in its application to the writing process, it remains an important initial model for understanding the sequence of psychological processes in these activities.

Providing accurate data on the process of creating advanced mathematics has proved notoriously difficult. Mathematicians are generally private individuals with a large cultural gap between their logical research paradigm and the paradigms of educational research (Nardi and lannone, 2004). Apart from introspective reports like Poincaré's (1908) perhaps the most noteworthy example of an account of actual advanced mathematical activity is that of Tall (1980) who was in the almost unique position of being a mathematician as well as a mathematics educator. Tall's approach could be described as analytical autoethnography in that he was 'a full member in the research group or setting, visible as such a member in the researcher's published texts, and committed to an analytic research agenda focused on improving theoretical understandings of broader social phenomena' (Anderson, 2006, p.375). The lack of any similar papers to Tall's since its publication, apart from Chick's (1998) deconstruction of her own thought processes in advanced mathematics, indicates that such an approach is difficult for mathematicians to emulate as they generally lack the ability to qualitatively reflect on and interpret the significance of their actions.

Rather than expecting mathematicians to qualitatively reflect on their own experiences or mathematics educators to capture advanced mathematical activity as it occurs, I propose that a more practical solution is to develop techniques by which mathematicians can 
capture their process of creating mathematics without distracting their concentration but not necessarily expect them to analyse it themselves. In this paper I present four techniques which I have used in the process of creating and writing up advanced mathematics. These techniques can be mapped onto different stages in the mathematics and writing creativity process, which I view as generally sequential, as shown in Table 1. Finally, I argue that the way mathematics research is communicated in journals should provide a means for mathematicians to present their process data as well as the product of their research which could then be analysed by mathematics educators or cognitive psychologists.

Table 1. Data capturing techniques for advanced mathematics and their relationship with the different stages of the mathematical creativity and writing processes.

\begin{tabular}{|l|l|l|l|}
\hline Process & Stage & Data capture technique & Reference \\
\hline \multirow{4}{*}{$\begin{array}{l}\text { Mathematical } \\
\text { creativity }\end{array}$} & Preparation & Proof plan & (Wolska et al., 2004) \\
\cline { 2 - 4 } & Incubation & Concept map & $\begin{array}{l}\text { (Bolte, 1999; Lavigne et } \\
\text { al., 2008) }\end{array}$ \\
\cline { 2 - 4 } & Illumination & Activity transcript & (Tall, 1980) \\
\cline { 2 - 4 } & Verification & & \\
\hline \multirow{4}{*}{ Writing } & Preparation & & (Margerum-Leys, 1999) \\
\cline { 2 - 4 } & Incubation & Concept map & \\
\cline { 2 - 4 } & Writing & & $\begin{array}{l}\text { Annotated draft and } \\
\text { transcript }\end{array}$ \\
\cline { 2 - 4 } & Revision & & (Eliot, 1971) \\
\hline
\end{tabular}

\section{The data capturing techniques}

The four data capturing techniques are described in the order that they might be used in the production of a mathematical research article. Each technique has been illustrated by an example from my own $\mathrm{PhD}$ research work. Apart from illustrating these techniques, the second and fourth examples were chosen because their content appeared to be interesting from more than a mathematical perspective: the former contains an account of making a mistake, getting stuck and then overcoming it; the latter indicates my emotional state when reviewing my research writing and my struggles to remember something I had done in the past. 


\section{Proof planning}

This first data capture technique proposed is proof planning. This term was probably originally used by Wallen (1983) in computational mathematics to describe automated theorem proving techniques and is now commonly used in this field. However, little research has been carried out to compare computational proof planning with the actual proving methods of mathematicians (although, see Wolska et al., 2004). It is used here to describe the process of creating an informal plan for a proof. An example of its use in advanced mathematics is provided in Figure 1. I created this plan in preparation for a meeting with my PhD supervisor. It is likely that this technique will be motivated by some kind of social interaction for the plan to be written down. In this case, the structure of the actual final proof was remarkably similar to the plan.

Figure 1. Part of plan for a proof that a one dimensional unsteady wave equation is an example of a cusp catastrophe.

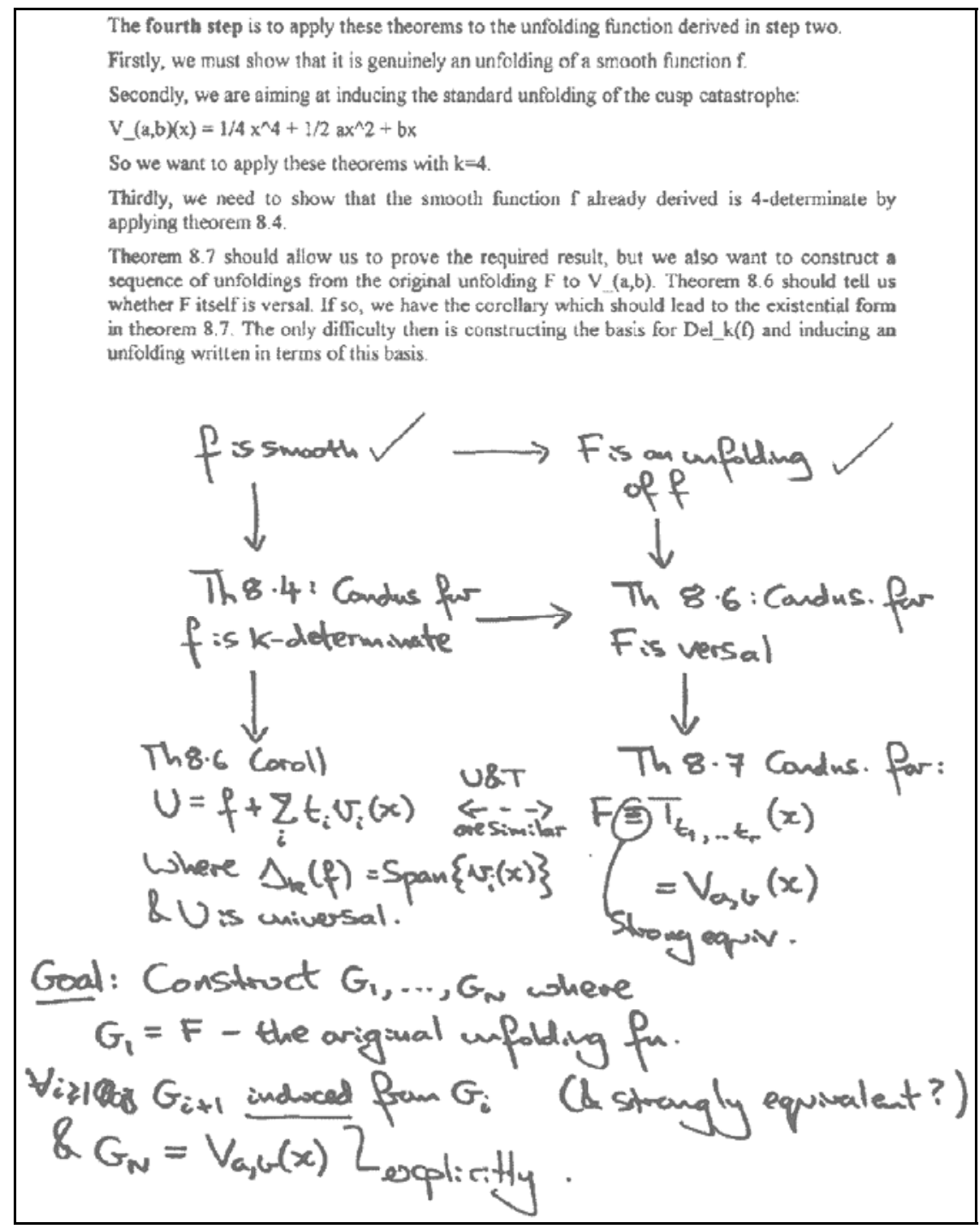




\section{Activity transcripts}

An activity transcript is an account of an episode of mathematical activity. It combines notes from the mathematical activity with a journal style account. Journal writing has been suggested for undergraduate mathematics classes (Rosenthal, 1995) but is unusual in more advanced mathematics research. An example of an activity transcript for advanced mathematics is given in Table 2. It is divided into four parts: a background to the mathematical activity (written eight days after it took place), notes from the activity itself, a written-up version of these notes (also written after eight days) and reflections on the activity (written after three weeks and written up after two and a half months). The time delay in writing up a mathematical activity appears to be important but an appropriate length of time may depend upon the writer's context and personal preferences. This approach is similar to Tall's (1980) article but it records a single mathematical activity in more detail and does not include qualitative analysis of the significance of the experience. It is therefore more practical for a mathematician to use if they wish to write up a single episode of mathematical activity which may include mistakes, such as the one shown in Table 2.

\section{Concept maps}

Concept maps are:

Graphical tools for organizing and representing knowledge. They include concepts, usually enclosed in circles or boxes of some type, and relationships between concepts indicated by a connecting line linking two concepts. Words on the line, referred to as linking words or linking phrases, specify the relationship between the two concepts. (Novak and Cañas, 2008, p.1)

Bolte (1999) has proposed the use of concept maps for assessment in undergraduate mathematics courses. They have also been used as a research tool by Lavigne and others (2008) for exploring students' understanding of undergraduate statistics. Margerum-Leys (1999) promotes their use as an aid to understanding in the pre-writing stage of the writing process. Figure 2 provides a concept map for a chapter of one of my mathematics research reports (Samuels, 1989) on the method of characteristics applied to unsteady wave equations. 


\section{Table 2. Four extracts from an example activity.}

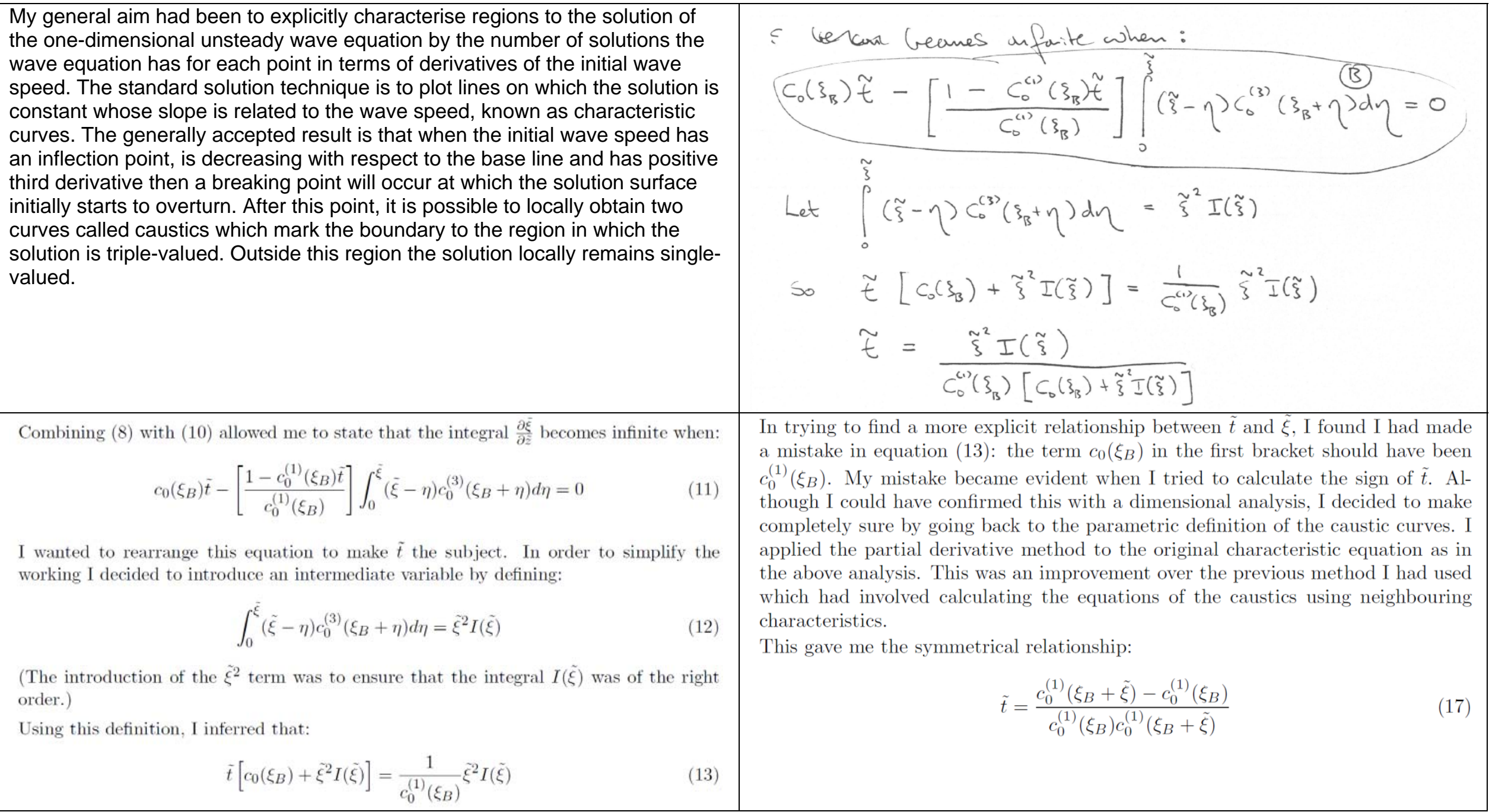


Figure 2. Concept map of the method of characteristics applied to unsteady wave equations (produced using Inspiration ${ }^{\circledR}-$ see http://www.inspiration.com/).

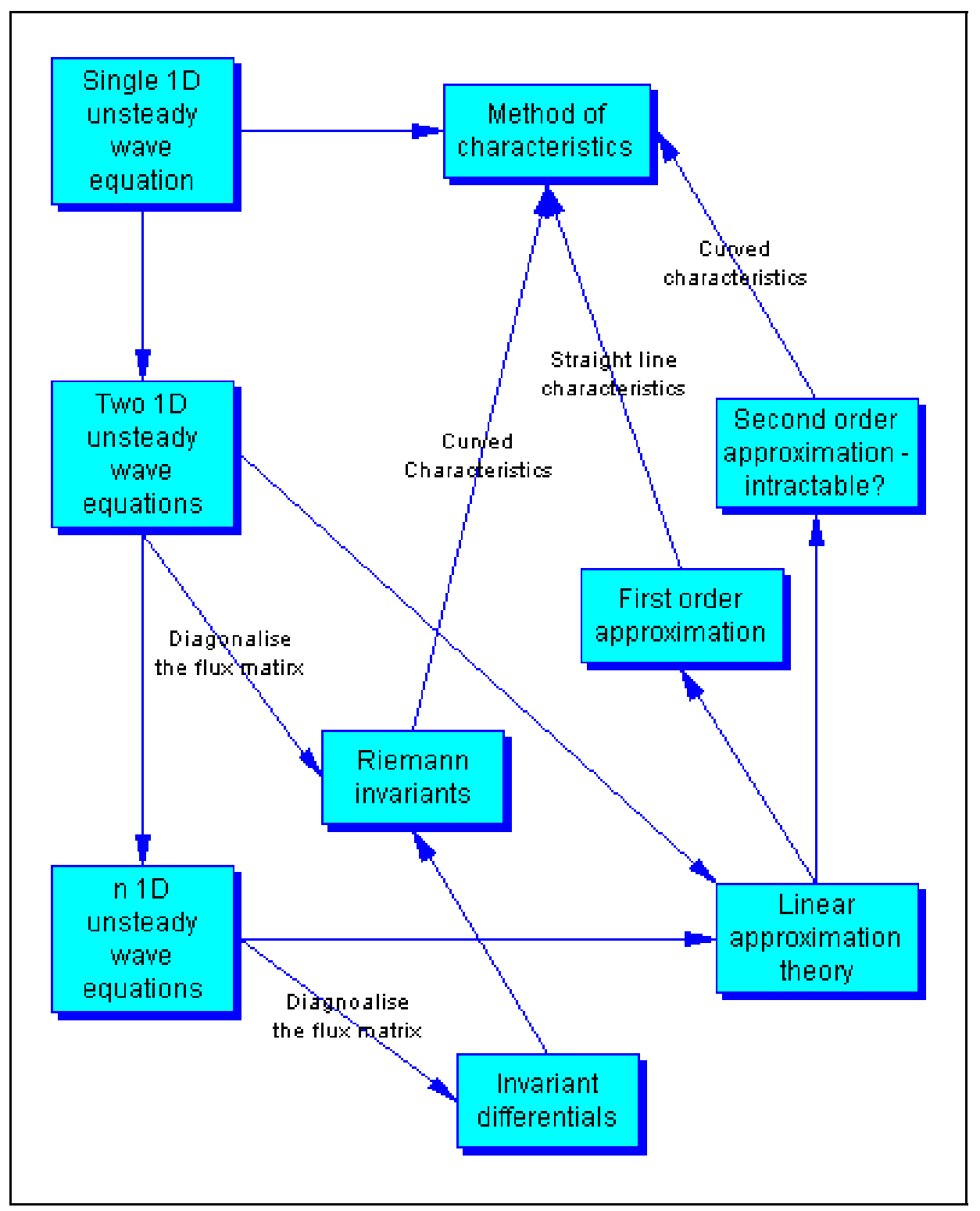

\section{Annotated draft and transcript}

The final data capturing technique proposed here is an annotated draft and transcript. It is similar to the approach used by Valerie Eliot to represent the annotated draft of her late husband's poem, The Waste Land (Eliot, 1971). I developed this technique as a means to capture my 'thinking aloud' as I re-read my internally published research reports. An example of an annotated draft of an extract from Samuels (1989) with its transcription is given in Figures 3 and 4. 


\section{Figure 3. Facsimile of a research report with 'thinking aloud' comments.}

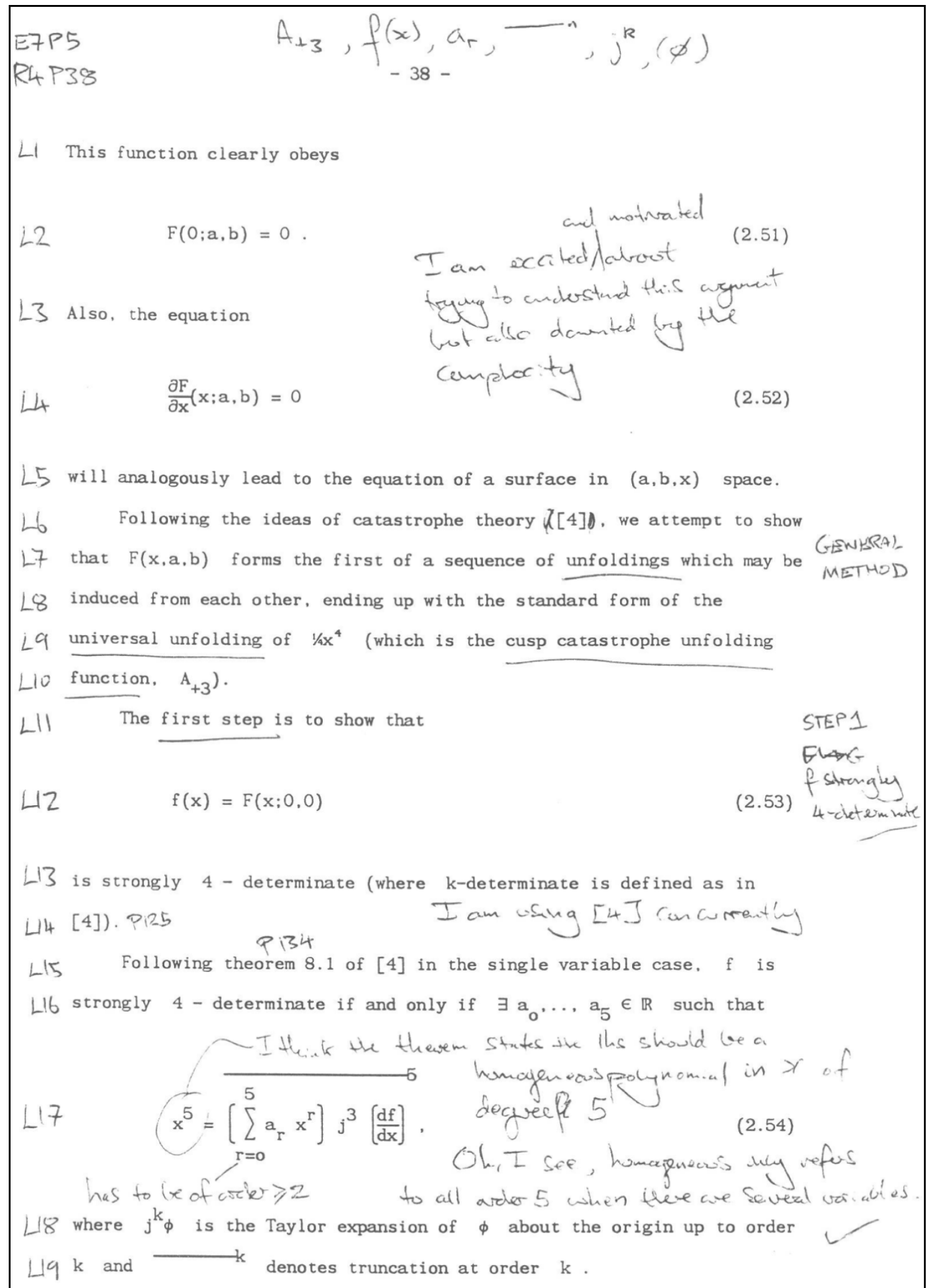




\section{Figure 4. Transcript of the 'thinking aloud' comments on the extract in Figure 3.}

Note:

1. 'Top' refers to the top of the page - this is a list of variables defined on this page which indicates the short term memory load for the reader.

2. ' $L n$ ' refers to line number $n$.

3. Text to the left of the colon in bold is a positional descriptor for an action or an annotation.

4. Words in italics describe an action.

5. Words in normal font are an annotation.

\section{Exract 7 page 5}

Report 4 page 38

Top: $A_{+3}, f(x), a_{r}, \stackrel{n}{n}, j^{k},(\phi)$

L1-L4: I am excited ('and motivated' inserted) about trying to understand this argument but also daunted by the complexity

L6, ([4]): brackets removed

L7, unfoldings: underlined

L7: GENERAL METHOD

L9, universal unfolding: underlined

L9-L10, cusp catastrophe unfolding function: underlined

L11, first step: underlined

L11: STEP 1

$(F \rightarrow G)$ crossed out

$f$ strongly 4-determinate

L14: P125

L13-L14: I am using [4] concurrently

L15, theorem 8.1: P134

L17, $x^{5}$ : I think the theorem states that lhs should be a homogeneous polynomialin $x$ of degree ('f' crossed out) 5

Oh, I see, homogeneous only refers to all order 5 when there are several variables.

L17, $\sum_{r=0}^{5} a_{r} x^{r}$ : has to be of order $\geq 2$

L18: ticked 


\section{Discussion}

In this discussion paper I have presented four data capturing techniques for advanced mathematical activities with examples of each from my own research. I have not attempted to analyse the data provided through these techniques as I believe this would be done more appropriately and objectively by another educational or cognitive psychologist researcher. However, as explained above, there was an additional non-mathematical motivation behind including the second and fourth examples. Rather, they exemplify how a mathematician might present content laden data, with each technique potentially providing insight into their thought processes when carrying out advanced mathematics research as well as conveying their mathematical meaning. These techniques are easy to learn and are not time consuming to use. Their use may actually enhance mathematical activity and the process of writing it up rather than detract from it, overcoming mathematicians' sense of alienation from the production of mathematics research.

I propose the establishment of a corpus of advanced mathematical process data, similar to the Digital Variants corpus (Björk, 1998, see http://www. digitalvariants.org/) where mathematicians are able to supply their content laden data of different stages of the mathematics creativity process and the process of writing it up, and other researchers are able to analyse their thought processes. Alternatively, this could partially be achieved by using the additional online resources facility available with some journals.

\section{References}

Anderson, L. (2006) 'Analytic autoethnography', Journal of Contemporary Ethnography, 35(4), pp. 373-395.

Björk, S. and Holmquist, L. (1998) 'Exploring the Literary Web: The Digital Variants Browser', in Proceedings of Literature, Philology and Computers Conference, 7-9 September. Edinburgh.

Bolte, L.A. (1999) 'Using concept maps and interpretive essays for assessment in mathematics', School Science and Mathematics, 99(1), pp. 19-30. 
Chick, H. (1998) 'Cognition in the formal modes: research mathematics and the SOLO taxonomy', Mathematics Education Research Journal, 10(2), pp. 4-26.

Crowley, S. (1977) 'Components of the composing process', College Composition and Communication, 28(2), pp. 166-169.

Eliot, T.S. (1971) The waste land: a facsimile and transcript of the original drafts including the annotations of Ezra Pound. London: Faber and Faber, Edited by V. Eliot.

Flower, L. and Hayes, J.R. (1981) 'A cognitive process theory of writing', College Composition and Communication, 32(4), pp. 365-387.

Guilford, J.P. (1950) 'Creativity', American Psychologist, 5(9), pp. 444-454.

Lavigne, N.C., Salkind, S.J., and Yan, J. (2008) 'Exploring college students' mental representations of inferential statistics', Journal of Mathematical Behavior, 27(1), pp. 11-32.

Lubart, T.I. (2001) 'Models of the creative process: past, present and future', Creativity Research Journal, 13(3-4), pp. 295-308.

Margerum-Leys, J. (1999) Concept mapping as a prewriting activity: a presentation for MACUL 99. Available at: http://www-personal.umich.edu/ -imargeru/conceptmap/ (Accessed: 25 April 2012).

Marx, K. (1974) 'Estranged Labour', in Marx, K. The economic and philosophical manuscripts of 1844. $4^{\text {th }}$ Edition. Moscow: Progress Publishers, pp. 61-74.

Nardi, E. and lannone, P. (2004) 'On the fragile, yet crucial, relationship between mathematicians and researchers in mathematics education', Proceedings of the 28th Conference of the International Group for the Psychology of Mathematics Education, 3, pp. 401-408. 
Novak, J.D. and Cañas, A.J. (2008) The theory underlying concept maps and how to construct and use them, Technical Report IHMC CmapTools 2006-01 Rev 01-2008, Florida Institute for Human and Machine Cognition. Available at:

http://cmap.ihmc.us/Publications/ResearchPapers/TheoryUnderlyingConceptMaps. pdf (Accessed: 25 April 2012).

Poincaré, H. (1908) 'L'invention mathématique', Bulletin de l'Institut Général de Psychologie, 8e année (3), pp. 175-196.

Rosenthal, J.S. (1995) 'Active learning strategies in advanced mathematics classes', Studies in Higher Education, 20(2), pp. 223-228.

Rowan, J. (1981) 'A dialectical paradigm for research', in Reason, P. and Rowan, J. (eds.) Human inquiry: a sourcebook of new paradigm research. Chichester: John Wiley and Sons, pp. 93-112.

Samuels, P.C. (1989) Shock behaviour and diffusion. Technical report 12/89, Department of Mathematics. University Reading.

Tall, D.O. (1980) 'The anatomy of a discovery in mathematics research', For the Learning of Mathematics, 1(2), pp. 25-34.

Wallen, L. (1983) Using proof plans to control deduction. University of Edinburgh, Division of Informatics, Research Paper no. 185. Available at: http://www.dai.ed.ac.uk/pub/daidb/papers/rp185.pdf (Accessed: 25 April 2012).

Wolska, M., Vo, B.Q., Tsovaltzi, D., Kruijff-Korbayová, I., Karagjosova, E., Horacek, H., Fiedler, A., and Benzmüller, C. (2004) 'An automated corpus of tutorial dialogs on mathematical theorem proving', in Lino, M.T., Xavier, M.F., Ferreira, F., Costa, R. and Silva, R. (eds.) Proceedings of the 4th International Conference on Language Resources and Evaluation (LREC-04). Paris: ELRA, pp. 1007-1010. 


\section{Author details}

Dr Peter Samuels is an Academic Skills Tutor at Birmingham City University, UK, and a Visiting Research Fellow at the Serious Games Institute at Coventry University, UK. His research interests include university-level mathematics education (especially the use of new technologies and serious games) and learning development. 